Supporting Information for:

\title{
Solvent and Solvent Isotope Effects on the Vibrational Cooling Dynamics of a DNA
}

\section{Base Derivative}

Chris T. Middleton, Boiko Cohen, and Bern Kohler

Assignment of the Initial Vibrational Temperature. A vibrational temperature can be assigned to the molecule by assuming that Boltzmann-distributed populations are present in all $3 \mathrm{~N}-6$ normal modes of a nonlinear polyatomic molecule. If the vibrations can be described by separable normal modes with harmonic oscillator frequencies $v_{i}$, then the vibrational energy of a nonlinear, polyatomic molecule with $N$ atoms in excess of its zero-point energy at temperature $T$ is given by,

$$
E_{v i b}(T)=\sum_{i=1}^{3 N-6} h v_{i}\left[\exp \left(\frac{h v_{i}}{k_{B} T}\right)-1\right]^{-1}
$$

The vibrational temperature, $T$, of the hot ground state molecules is obtained by solving for the value of $T$ that makes $E_{v i b}(T)$ equal to the sum of the vibrational energy prior to photoexcitation, $E_{v i b}\left(T_{0}\right)$, and the excess vibrational energy remaining at time $t . T_{0}$ is the temperature of the solute-solvent system prior to excitation of the solute, and is equal to $295 \mathrm{~K}$ in our experiments. The initial vibrational temperature, $T_{1}$, produced immediately after internal conversion and prior to any significant intermolecular energy transfer is obtained by solving eqn. 2 ,

$$
E_{v i b}\left(T_{1}\right)=E_{v i b}\left(T_{0}\right)+\frac{h c}{\lambda_{e x c}}
$$


The second term in eqn. 2 is the excess vibrational energy produced by ultrafast decay to $\mathrm{S}_{0}$ after excitation with a photon of wavelength $\lambda_{e x c}$. Equating the photon energy with the excess vibrational energy neglects solvation and other energy relaxation mechanisms that could occur prior to the establishment of Boltzmann-distributed vibrational populations. Because internal conversion occurs at a much greater rate than vibrational cooling, we assume there is negligible energy loss to the solvent during the former process. In this case, the entire energy of each $267 \mathrm{~nm}$ pump photon $\left(\approx 37000 \mathrm{~cm}^{-1}\right)$ is deposited into vibrational modes of $\mathrm{S}_{0}$. Using calculated normal mode frequencies ${ }^{1}$ and an excitation wavelength of $267 \mathrm{~nm}, T_{1}$ is calculated to be $1850 \mathrm{~K}$ for $9 \mathrm{MA}$ and $1831 \mathrm{~K}$ for $9 \mathrm{MA}-d_{2}$.

\section{Supporting References}

(1) Xue, Y.; Xie, D. Q.; Yan, G. S. International Journal of Quantum Chemistry 2000, 76, 686. 